APDS: The Autonomous

Pathogen Detection System

Benjamin Hindson, Anthony Makarewicz, Ujwal Setlur, Bruce Henderer, Mary McBride, John Dzenitis

October 6, 2004

Biosensors and Bioelectronics 
This document was prepared as an account of work sponsored by an agency of the United States Government. Neither the United States Government nor the University of California nor any of their employees, makes any warranty, express or implied, or assumes any legal liability or responsibility for the accuracy, completeness, or usefulness of any information, apparatus, product, or process disclosed, or represents that its use would not infringe privately owned rights. Reference herein to any specific commercial product, process, or service by trade name, trademark, manufacturer, or otherwise, does not necessarily constitute or imply its endorsement, recommendation, or favoring by the United States Government or the University of California. The views and opinions of authors expressed herein do not necessarily state or reflect those of the United States Government or the University of California, and shall not be used for advertising or product endorsement purposes. 


\section{APDS: The Autonomous Pathogen Detection System}

\section{UCRL-JRNL-207074}

Benjamin J. Hindson, Anthony J. Makarewicz, Ujwal S. Setlur, Bruce D. Henderer, Mary T. McBride, and John M. Dzenitis*

Lawrence Livermore National Laboratory, 7000 East Avenue, P.O. Box 808, Livermore, CA 94550

*Corresponding Author:

John M. Dzenitis

Lawrence Livermore National Laboratory

L-174, P.O. Box 808, 7000 East Avenue

Livermore, Ca 94551

(p) 925-422-6695

(f) $925-424-2778$

dzenitis2@1lnl.gov 


\begin{abstract}
We have developed and tested a fully autonomous pathogen detection system (APDS) capable of continuously monitoring the environment for airborne biological threat agents. The system was developed to provide early warning to civilians in the event of a bioterrorism incident and can be used at high profile events for short-term, intensive monitoring or in major public buildings or transportation nodes for long-term monitoring. The APDS is completely automated, offering continuous aerosol sampling, in-line sample preparation fluidics, multiplexed detection and identification immunoassays, and nucleic-acid based polymerase chain reaction (PCR) amplification and detection. Highly multiplexed antibody-based and duplex nucleic acid-based assays are combined to reduce false positives to a very low level, lower reagent costs, and significantly expand the detection capabilities of this biosensor. This article provides an overview of the current design and operation of the APDS. Certain sub-components of the ADPS are described in detail, including the aerosol collector, the automated sample preparation module that performs multiplexed immunoassays with confirmatory PCR, and the data monitoring and communications system. Data obtained from an APDS that operated continuously for seven days in a major U.S. transportation hub is reported.
\end{abstract}

\title{
Keywords
}

Detection, immunoassay, PCR, pathogen, APDS 


\section{Introduction}

One of the easiest methods of exposing a large population to a biological threat agent is through an aerosol; this was clearly evidenced by the anthrax attacks that occurred in 2001. Environmental monitoring technologies capable of rapidly detecting and identifying aerosols of multiple pathogens or toxins improve our ability to counter biological terrorism (Franz et al., 1997). An ideal aerosol monitoring system would be capable of detecting and identifying multiple pathogens in real or near-real time. The system should run unattended for long periods of time, require infrequent maintenance, and be inexpensive to operate. The platform should utilize detection methodologies with sensitivities adequate to detect life-threatening doses of airborne pathogens (Hilleman, 2002), and with sufficient selectivity to virtually eliminate false positives. Since 2001, considerable research effort has been directed toward the development of biological detection and identification technologies that meet these criteria (Luoma et al, 1999; Stratis-Cullum et al., 2003; Cheng et al., 1999; Snyder et al., 1999; Sincock et al., 1999; Ryzhov et al., 2000) and several reports describe autonomous aerosol collection followed by in-line sample processing with identification and quantification (Fitch et al., 2003; Anderson et al., 1999; Ligler et al., 1998; Lester and Ponce, 2002; Makino et al., 2002; Donlon and Jackman, 1999).

We have developed, built and tested the Autonomous Pathogen Detection System (APDS), a standalone instrument capable of continuous, fully autonomous monitoring for multiple airborne biological threat agents (Dzenitis et al., 2003). The APDS is intended for use in domestic applications (e.g., office complexes, transportation terminals) where the public is at high risk of exposure to covert releases of bioagents, and as part of an integrated network of biosensors for wide-area monitoring of urban areas and major gatherings (e.g., inaugurations, Olympics). The APDS continuously collects aerosol samples, performs multiplexed antibody-based tests (immunoassays) at 60 minute intervals, performs automated real-time flow-through nucleic acid assays (PCR) testing to confirm an immunoassay screen reactive, 
archives samples, conducts data reporting and silent alarming with continuous unattended operation benchmarked at 7 days. Multiplexed immunoassays allow the detector to respond to broad classes of bioagents, including those without nucleic acids such as protein toxins, and are used to reduce reagent costs, making long term monitoring operations possible. PCR provides a secondary, confirmatory assay. By utilizing two highly specific biological assays that rely on fundamentally different molecular interactions for detection, the probability of reporting false alarms is greatly reduced. The system is contained in a rugged, mobile chassis so the only utilities required are AC power. A Labview-based software program controls instrument functions and performs data acquisition, real-time data analysis, and result reporting. The fully autonomous aerosol collection and sample preparation capabilities limit maintenance requirements making integration into a central security or monitoring network possible.

We have evaluated the performance of the fully-integrated APDS in two end-to-end systems tests conducted in specialized aerosol chambers (McBride et al., 2003; Hindson et al., 2004), and the system has conducted thousands of assays in a variety of real-world deployment scenarios. Here, we describe the components of the system, and discuss some of the operational performance parameters in greater detail. Multiplexed immunoassay and real-time PCR data from an APDS that operated continuously for seven days in a major U.S. transportation hub is presented. 


\section{Instrument}

\subsection{Concept of operation}

A process flow diagram for environmental monitoring with the APDS is shown in Fig. 1. The APDS continuously collects particulates from the air into water. Once an hour, the aerosol collector dispenses a liquid sample to the automated fluidics module. The fluidics module performs a multiplexed immunoassay and dispenses the processed sample to the detector for analysis. The detector (flow cytometer) performs the analysis, and the result is subsequently processed using a triggering algorithm. If the immunoassay result is negative, the aerosol collector dispenses the next sample and the process is repeated. An immunoassay result for any agent that is above the detection threshold set for that agent is considered "reactive" and will result in a notification page being sent to Lawrence Livermore National Laboratory (LLNL) personnel (middle column). If the immunoassay result is reactive and the agent contains DNA, a confirmatory PCR is initiated (indicated in the column on the right). An aliquot of the original sample is taken for the PCR assay and mixed with the PCR reagents. Once PCR is underway, the aerosol collector dispenses the next sample, and the next immunoassay is started. Most of the time, the system will operate in the column shown on the left. Each of the components that make up the APDS is described in more detail below. 


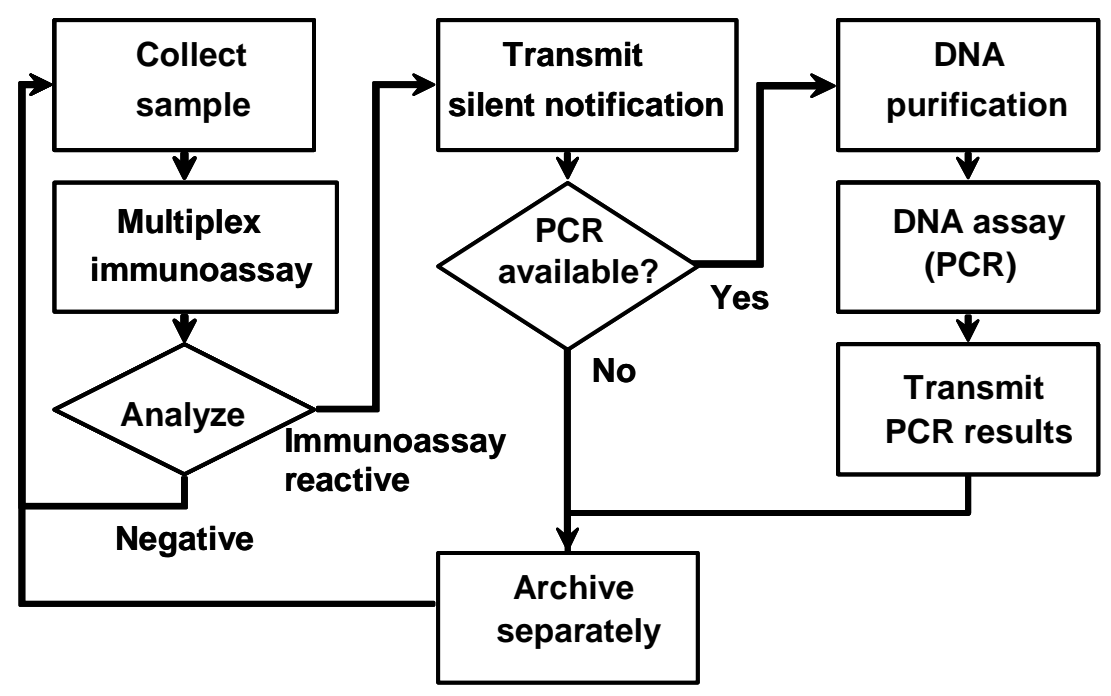

Fig. 1. Process flow diagram for environmental monitoring with the APDS, including confirmatory PCR. Most of the time the system operates in the left column. A potential immunoassay screen reactive sends the system to the second (middle) loop. This response represents both actions that may be taken by the instrument itself (e.g., paging experts with a request for data review) and by external systems (e.g., security cameras being checked). If the agent contains DNA, then PCR confirmation is initiated. A screen reactive PCR test results in a more extensive response.

\subsection{Aerosol collector}

Because aerosol collectors containing dry matrix-type filters are difficult to couple to autonomous systems and are relatively non-selective in the types and sizes of particles collected, we developed a twostage aerosol collector that utilizes an LLNL-designed virtual impactor pre-concentration stage in front of a commercial wetted-wall cyclone collector (Smart Air Sampler System (SASS) 2000 ${ }^{\mathrm{TM}}$, Research International, Woodinville, WA) (Fig. 2). For biological agents to be effective weapons, they must be disseminated as aerosols consisting of particles $(0.5-5 \mu \mathrm{m})$ that can be inhaled and retained in the human lung (Eitzen et al., 1999). The virtual impactor has been optimized to capture particles in the 1-10 $\mu \mathrm{m}$ size range and the wetted-wall cyclone traps the particles in fluid, making downstream processing much easier. 
The virtual impactor separates particles based on momentum. Entering air is split into two flow streams; the bypass (or major) flow and the product (or minor) flow. The ratio of the bypass to product flow is at least 4:1 and as much as 9:1. The bypass flow is turned sharply from the direction of the entering air flow, while the product flow traces a straight path relative to the entering flow, albeit at a much lower velocity. Consequently, large particles with high momentum follow a straight path and enter the product flow, whereas small particles make the turn into the bypass flow. The product flow enters a wetted wall cyclone, where the flow makes a 90 degree turn and spirals up a cylindrical plastic pipe. Inside the cyclone, a curtain of water is pulled up the walls of the cyclone due to the air flow. Particles within the cyclone either impinge a sheet of water at the cyclone inlet or move to the wetted wall due to centripetal force. Either way, they are captured in the water volume. Water pulled up the walls by the air flow circulates through the cyclone, collecting in a cistern near the top of the cyclone, and is returned to the bottom of the cyclone via a small recirculation tube. An optical detector is integral to the recirculation tube, monitoring the air/water interface passing through the recirculation tube. The number and speed of the interfaces is correlated to total water volume within the cyclone. When the water volume falls below a set level, a valve opens to a pressurized water tank, allowing about $0.5 \mathrm{ml}$ of water to enter the cyclone.

The APDS/SASS 2000 hybrid collector can sample up to 3000 liters of air per minute (lpm), allowing many more particles to be collected over a shorter period (the SASS 2000 samples about 275 lpm). An on-board computer controls air flow rates and the size range of particles collected. Particle collection, fractionation and concentration are nearly continuous in autonomous operation. At prescribed intervals, the collector pauses and a peristaltic pump delivers the collected fluid to the sample preparation module. Aerosol collection resumes immediately, and at the same time, sample processing begins. 


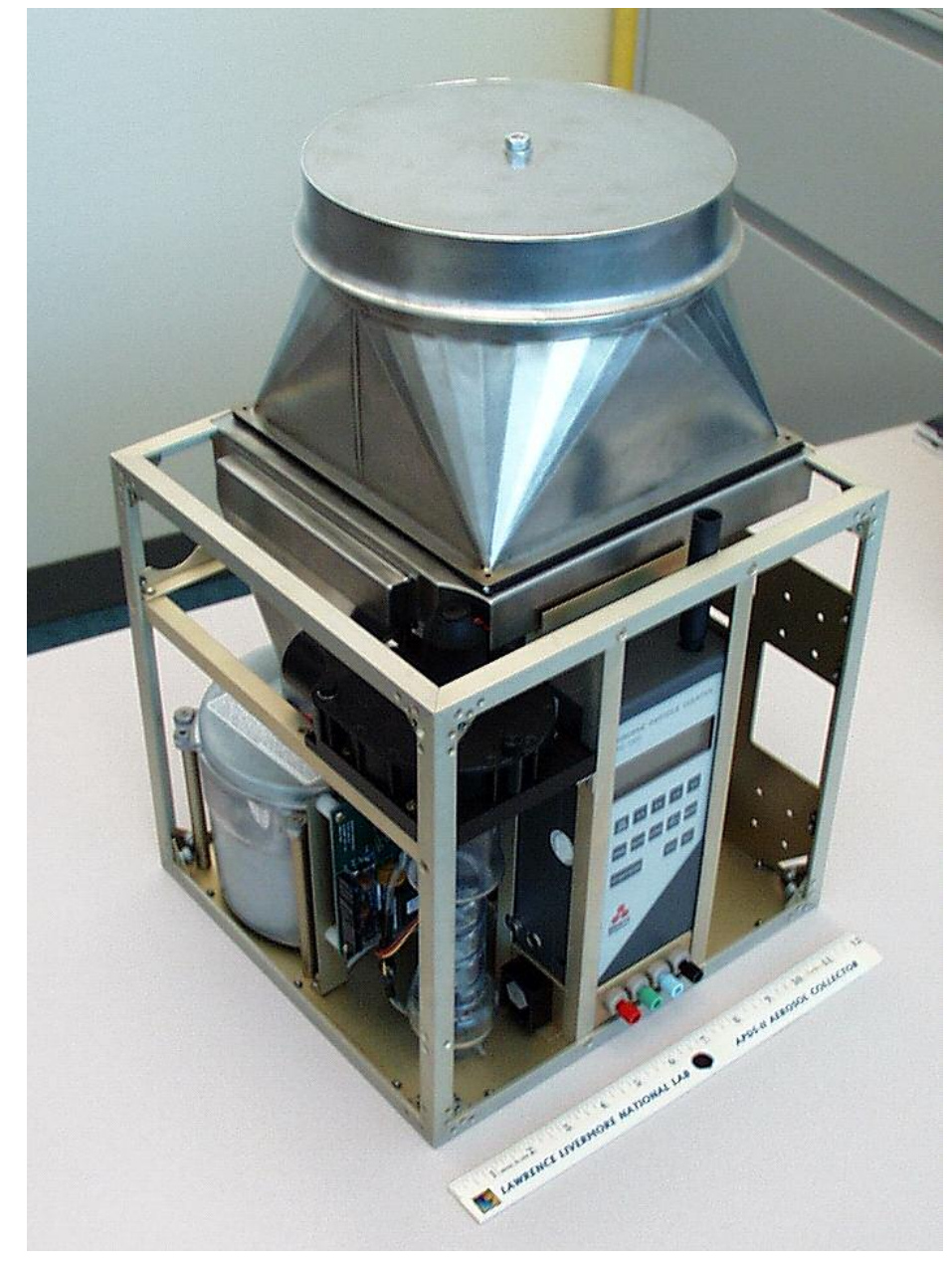

Fig. 2. The LLNL/SASS 2000 hybrid aerosol collector. The stack and cap can be seen at the top of the device. The virtual impactor is hidden below the stack. The large silver blower (back left) is the bypass fan and the small black fan is the product fan. The clear plastic cyclone is seen beneath the product fan.

\subsection{Sample preparation module}

The sample preparation (fluidics) module utilizes a powerful, highly flexible technique called sequential injection analysis (SIA) (Lenehan et al., 2002). The commercially available SIA system (FloPro, ${ }^{\mathrm{TM}}$ Global FIA, Gig Harbor, WA) reproduces functions routinely performed by lab personnel on the bench: moving the sample from the aerosol collector, preparing the sample (mixing, filtering, incubation, 
etc.), and delivering the sample reaction volume to the assay detectors. The SIA system (Fig. 3) comprises two bi-directional syringe pumps, two holding coils, four multi-position selection valves and a computer.

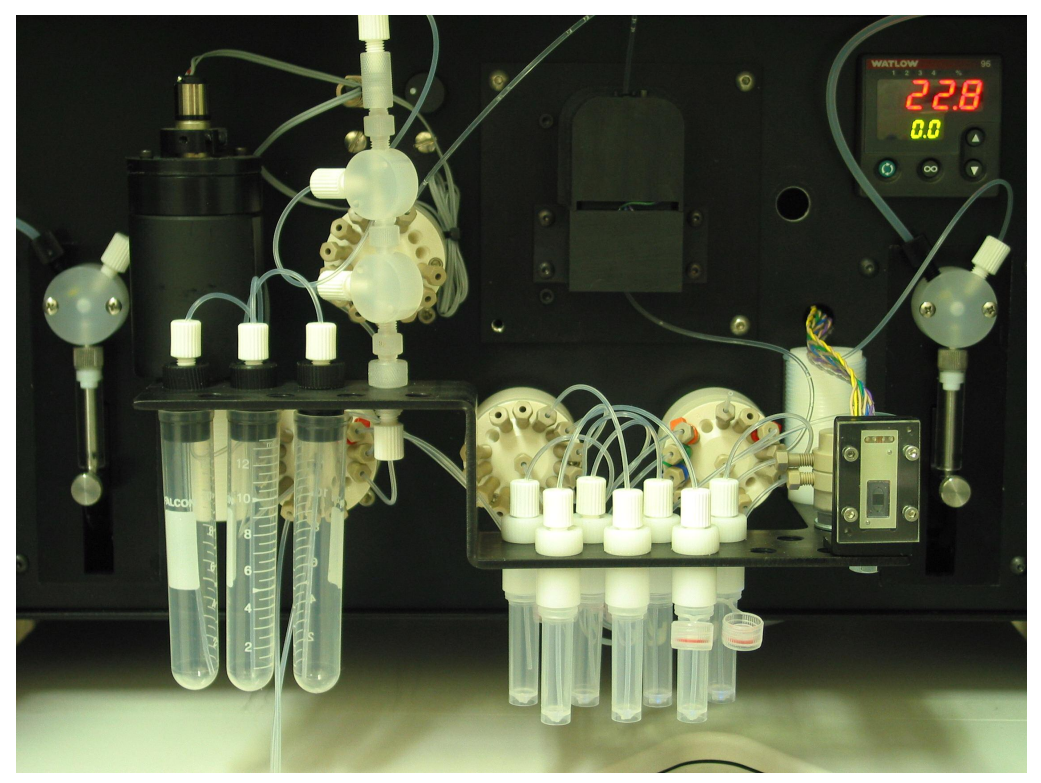

Fig. 3. The APDS sample preparation module. Sequential injection analysis (SIA) enabled simple, versatile, and reliable automated fluid handling. The integrated module is capable of conducting both automated multiplexed immunoassays and duplex flow-through PCR. The module consists of two bidirectional syringe pumps, two holding coils, and four 14 port multi-position selection valves. The immunoassay specific components (left side of photograph) include several reagent reservoirs and a microsphere sequestering cell. The PCR specific components (right side of photograph) include various reagent reservoirs, a thermal-cycler (upper right), two-channel (FAM/TAMRA) optical platform for realtime fluorescence detection, and a micro fabricated pillar chip for DNA extraction.

\subsection{Multiplexed immunoassay detection}

We have developed immunoassays that are rapid, sensitive, specific and can detect more than one threat agent simultaneously (i.e., multiplexed) from a single sample (McBride et al., 2003). The assays 
have been developed for use with a commercially available flow cytometer, the Luminex ${ }^{\mathrm{TM}}$ LX-100 (Luminex Corp, Austin, TX). The Luminex platform offers a 100 channel array, that utilizes surfacefunctionalized polystyrene microspheres $(5.6 \mu \mathrm{m})$ embedded with unique ratios of two different fluorophores. Potentially, up to 100 different analytes can be measured simultaneously within a single sample. The APDS employs a sandwich immunoassay format, where microspheres functionalized with antigen-specific capture antibodies are incubated with antigen, and then bound analyte is detected using biotinylated antibodies labeled with the fluorescent reporter, streptavidin-phycoerythrin (SA-PE). Each optically-encoded and fluorescently-labeled microsphere is then interrogated by the flow cytometer. Classification $(635 \mathrm{~nm})$ and reporter $(532 \mathrm{~nm})$ lasers excite embedded and surface bound fluorophores, respectively. The flow cytometer interrogates hundreds of microspheres per second; analysis is complete in 60s.

Currently, the APDS multiplexed immunoassays screen each sample for up to 8 different pathogens simultaneously. In addition, every assay utilizes four inbuilt assay controls that convey important diagnostic information regarding reagent addition, quality and concentration, assay operator performance and instrument stability; controls can be easily added without compromising or limiting the screening capabilities of an assay. These assay controls have been described in previous publications (McBride et al., 2003; Hindson et al., 2004). Every sample is analyzed in the context of the performance of the controls, thereby minimizing the likelihood of false positives.

The fluidics manifold conducts automated immunoassays and real-time, confirmatory PCR using a series of simple fluidic manipulations (or sequences) designed to mimic the mechanics of the benchtop assays. The immunoassay portion of the fluidics manifold, in addition to the components described above, also employs reagent reservoirs, a microsphere sequestering cell (bead trap) where the wash assays are conducted; the module has been described in detail elsewhere (Hindson et al., 2004). 
Microspheres are placed in the sequestering cell and exposed, in turn, to sample, labeling antibodies, and the fluorescent reporter, SA-PE. Upon completion of the automated immunoassay, the fluidics module dispenses the processed sample to the flow cytometer for analysis. Data acquired by the Luminex is then analyzed using an algorithm. If the immunoassay results do not cause the algorithm to trigger, the remaining sample $(3.9 \mathrm{ml})$ is pumped to a common archive reservoir. The immunoassay fluidics components are cleaned and readied to accept the next sample from the aerosol collector. If the immunoassay results cause the algorithm to trigger, confirmatory PCR is called upon. The trigger instructs the PCR portion of the fluidics manifold to retrieve an aliquot from the original aerosol sample (the same sample used in immunoassay) and perform a DNA purification step followed by a confirmatory PCR test specific to the agent in question.

\subsection{DNA purification}

As PCR reactions are susceptible to interference from components present in the sample matrix (i.e., inhibited) we incorporated a DNA extraction step, using a "pillar chip", prior to PCR. The pillar chip is a micro-fabricated device made from a silicon wafer using standard photoresist/etching techniques. The chip has a well defined bed of pillars, with silica surfaces that are suitable for nucleic acid capture. Chips can be resistively heated and are prepared with two fluid connections. The device can accept a range of input volumes (100-1000 $\mu \mathrm{l})$ at different flow rates (1-100 $\mu \mathrm{l} / \mathrm{s})$. The performance of the chip has been detailed elsewhere (Visuri et al., 2002; Belgrader et al., 2002).

\subsection{Real-time, flow-through PCR detection}

The APDS uses a real-time TaqMan implementation of the polymerase chain reaction (PCR) (Nasarabadi et al., 1999; Fitch et al., 2002). Our PCR reaction mixture (master mix) comprises fluorescence resonance energy transfer (FRET) probes, forward and reverse primers to locate specific DNA sequences of interest, dNTPs, magnesium chloride, internal control DNA and the Taq polymerase 
enzyme. To realize duplexed PCR with our two channel fluorescence detector, the FRET probes incorporated 3' Black Hole Quencher and 5' FAM (agent) or TAMRA (internal control) modifications. The fluidics module assembles each PCR reaction, whereby sample ( $5 \mu$, potentially containing agent DNA) and master mix $(20 \mu \mathrm{l})$ are combined, pumped to the thermal-cycler then subjected to a temperature profile of $95^{\circ} \mathrm{C}$ for $60 \mathrm{~s}$, followed by 45 cycles of $95^{\circ} \mathrm{C}$ for $15 \mathrm{~s}, 60^{\circ} \mathrm{C}$ for $30 \mathrm{~s}$ and $72^{\circ} \mathrm{C}$ for $15 \mathrm{~s}$.

The PCR module consists of a thermal-cycler and a fluorescence detector and was based on an earlier version (Belgrader et al., 2003). The thermal-cycler has a flow-through chamber made from two photo-lithographically patterned and etched copper plates. The etched channel on the inside of the chamber allows the sample tubing to be clamped between the two chamber halves, ensuring good thermal contact. The tubing is connected directly to the fluidics system allowing the sample to be moved into and out of the thermal-cycler. The etched features on the outside of the chamber create increased surface area to enhance forced convective cooling. Heating is accomplished by clamping the chamber between two circuit board assemblies. Standard surface mount resistors soldered to the circuit boards act as heaters. A surface mount linear thermo-resistor provides temperature sensing for control of the thermal-cycling. During the cooling cycle, air is forced through slots in the circuit board assembly, onto the chamber.

The fluorescence detector has two independent channels, specific for FAM (excitation 480nm, emission 520nm) and TAMRA (excitation 520nm, emission 580nm) fluorophores. Apertures in copper plates create two optical paths perpendicular to the flow-through PCR tubing. For each channel, excitation light from a colored LED is filtered, and then focused onto the tubing which contains the PCR reagent plug. The resultant fluorescence emission is focused, filtered, then detected using a low-noise photodiode connected to an integrating chip. Each detection channel is operated independently. 
A control PCR reaction is performed twice daily, to ensure that the PCR module is functioning properly, that the PCR reagents have not changed or decayed, and that the samples being collected in any particular environment are not causing PCR reaction inhibition.

\subsection{Data monitoring and communications}

The communications sub-system provides transparent connectivity between an APDS in the field and a command console. Communication with fielded APDS units can be achieved via wireless (802.11b), cellular, or direct Ethernet wiring. The APDS command console (or a view-only remote console) allows immediate study of the instrument signals and performance at any time. An overall status map includes a map showing instrument locations, a list of instruments, and a timeline of instrument status, all color-coded. The colors have been selected to provide status at-a-glance. Any instrument on the status map can be clicked for a detailed status showing assay results over time and system data for that particular APDS unit. The remote console provides instant text paging specifying equipment problems or reactive screening results. The APDS sends out "Heartbeat" messages every 10 seconds. This message carries a status field and ensures connectivity. A "Data" message is sent out every minute, which contains status, state of the machine, and system parameters of interest. "Assay", "PCR" and "Error" messages are sent out when ever results are available. The command console sends out Command and Control messages as needed.

\subsection{Field testing and autonomous operation}

The performance of the APDS has been extensively tested in the laboratory, where the instrument has conducted many thousands of assays and the performance monitored through the use of spike tests, where agents were introduced into the liquid, assayed, and compared to our gold standard benchtop assays. We have evaluated the performance of systems that have been deployed (i.e., placed at off-site, strategic locations), where systems have operated continuously for days, weeks or months. We have 
collected more than 5000 samples from deployed systems, comprising over 30,000 assays; to date no false positives have been recorded. Fig. 4 shows multiplexed immunoassay and control duplex PCR data collected by an APDS that operated continuously for seven days in a major U.S. transportation hub. A total of 154 samples were analyzed by immunoassay, which represents 770 individual agent assays. The relative standard deviation of the antibody, fluorescence, instrument and negative controls were 3.9, 4.9, 5.9 and $16.8 \%$ respectively. All the immunoassay signals collected on the five agent channels were analyzed by our algorithm in real-time, none were reactive and confirmatory PCR was not triggered. A control PCR was executed twice daily over seven days, totaling 14 individual PCR reactions. No rise in fluorescence signal on the agent channel (FAM) was detected due to the absence of target DNA in the sample. The fluorescence signals on the internal control channel (TAMRA) rose consistently due to the presence of internal control DNA in the PCR master mix. The mean cycle threshold, calculated using an algorithm from a commercially available thermal-cycler, (Smart Cycler, Cepheid, CA) was 31.3 cycles with a relative standard deviation of $<1 \%$. These results indicate excellent repeatability and stability of the immuno- and PCR-based assays together with the associated instrumentation on which they were performed, during operation in a real-world environment. 
(a)
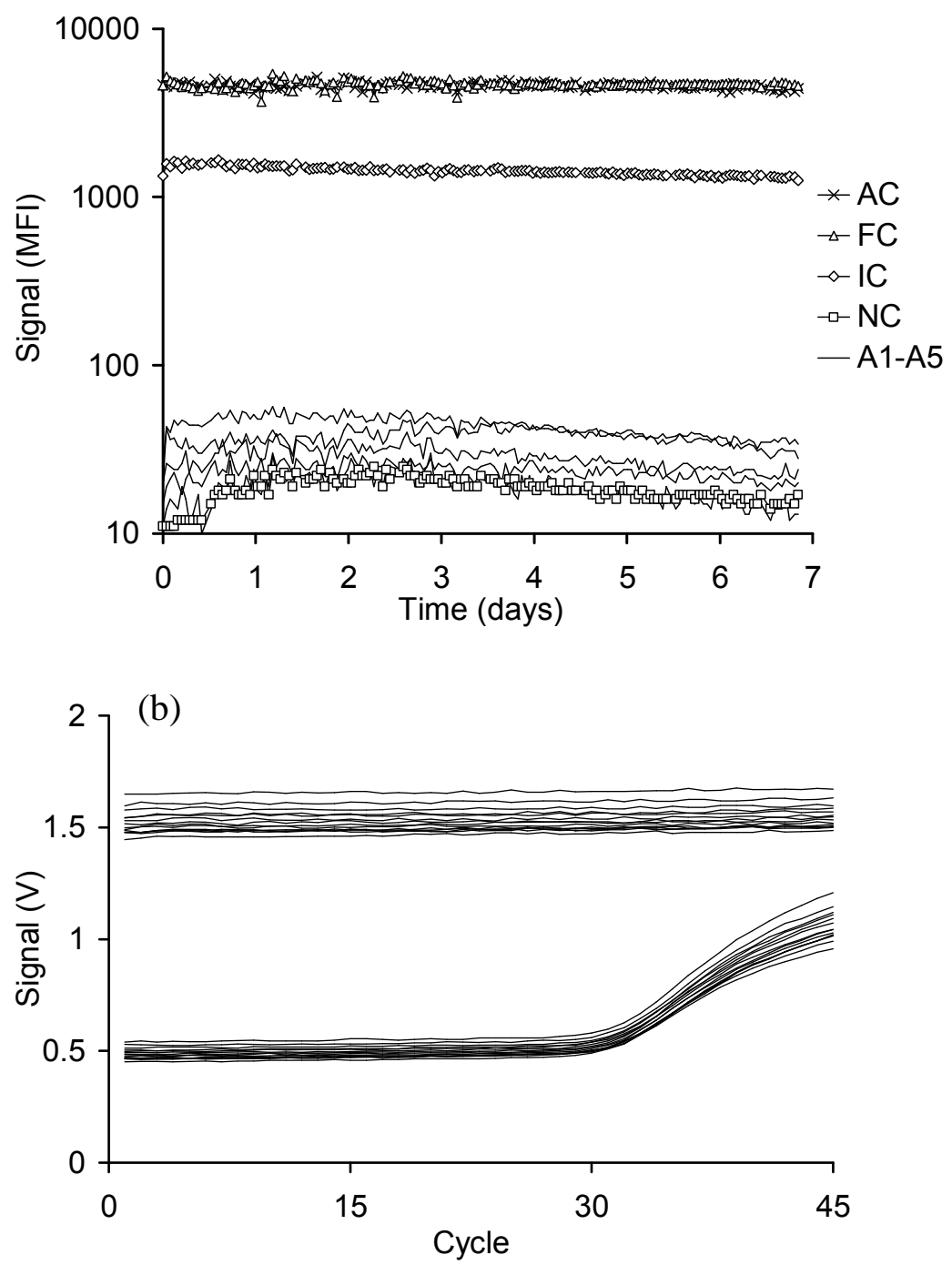

Fig. 4. Multiplexed immunoassay (a) and real-time, flow-through, duplex PCR data (b) from an APDS that operated for seven days in a major U.S. transportation hub. Samples were collected and analyzed hourly by immunoassay; the resultant immunoassay signals (MFI, median fluorescence intensity) are shown for four controls and five agents. A total of 154 samples were analyzed, which corresponds to 770 individual agent assays. Immunoassays signal designations are antibody control (AC), fluorescence control (FC), instrument control (IC), negative control (NC) and agents 1-5 (Ag 1-5). Real-time PCR was performed twice daily; a total of 14 consecutive control duplex PCR reactions are shown. Signals on the agent channel (FAM) remained flat in the absence of agent DNA in the sample, whilst those 
measured by the internal control channel (TAMRA) rose consistently after 31 cycles due to the presence of control DNA in the PCR reagent mixture.

The performance of the fully-integrated APDS has been evaluated in two end-to-end systems tests where the system was challenged with known concentrations of bioaerosols released in specialized containment chambers. The first chamber test was conducted in September, 2002 at Dugway Proving Grounds. The APDS with multiplex immunoassays was tested with live agent aerosols of Bacillus anthracis and Yersinia pestis. These tests proved that the end-to-end system could detect aerosolized, lethal bioagents (McBride et al., 2003). In September, 2003, we returned to Dugway Proving Grounds. The primary objective of the 2003 testing was to demonstrate the APDS with immunoassay identification and PCR confirmation of bacteria. A secondary objective was to demonstrate immunoassay identification of a protein toxoid (denatured toxin) aerosol release. Both testing objectives were achieved with multiple releases and clear identifications. The APDS was tested against, and shown to be effective for identifying aerosolized Bacillus anthracis, Yersinia pestis, Bacillus globigii, and botulinum toxoid. A total of 12 agent trials were conducted over 14 days of testing; the results of that test will be the subject of a subsequent report (Hindson et al., 2004).

\section{Conclusions}

We have developed and tested a fully autonomous pathogen detection system capable of continuously monitoring the environment for airborne biological threat agents. While the primary focus has been on protection of civilians from terrorist attacks, the same system could also have a role in protecting military personnel from biological warfare attacks. APDS instruments can be used at high profile events such as the Olympics for short-term, intensive monitoring or more permanent installation in major public buildings or transportation nodes. All of these units can be networked to a single command 
center so that a small group of technical experts could maintain and respond to alarms at any of the sensors. The APDS has several key advantages over competing technologies: (1) the ability to measure up to 100 different agents and controls in a single sample, (2) the flexibility and ease with which new microsphere-based assays can be developed and integrated into the system, (3) the presence of an orthogonal, real-time detection module for highly sensitive and selective nucleic acid amplification and detection, (4) the ability to use the same basic system components for multiple deployment architectures, and (5) the relatively low cost per assay $(<\$ 2.00$ per 10 -plex or $\$ 0.20$ per multiplexed immunoassay, with similar costs for the duplex PCR) and minimal consumables. The equipment cost of an APDS is higher than that of a dry filter collector currently used for environmental monitoring of biothreat agents the United States (Fitch et al., 2003). However, the APDS requires low labor which reduces operational costs over time, with much higher frequency reporting. Extensive validation of the APDS is still required, yet a significant burden of this task is reduced as the instrument utilizes Laboratory Response Network (LRN, Center for Disease Control) approved reagents.

\section{Acknowledgements}

The work presented here is the result of hard work by many individuals, many of whom were not listed in the author byline. Current and former APDS project team members who also participated in this work include (alphabetically): Bill Benett, Steve Brown, Keith Burris, Bill Colston, George Dougherty, J. Patrick Fitch, Dora Guiterrez, Dean Hadley, Richard Langlois, Don Masquelier, Tom Metz, Fred Milanovich, Shanavaz Nasarabadi, Sally Smith and Kodumudi (Venkat) Venkateswaran. We are grateful to our many collaborators who have helped make the project successful, including (but not limited to) Luminex, Inc., Global FIA, Tetracore, Inc., and our friends at Dugway Proving Grounds. This work was performed under the auspices of the U.S. Department of Energy by the University of California, Lawrence Livermore National Laboratory under Contract No. W-7405-Eng-48, with funding 
formerly from the DOE-CBNP Program, and currently through the Department of Homeland Security (DHS).

\section{References}

Anderson, G.P., King, K.D., Cuttino, D.S., Whelan, J.P., Ligler, F.S., MacKrell, J.F., Bovais, C.S., Indyke, D.K., Foch, R.J., 1999. Biological agent detection with the use of an airborne biosensor. Field Analyt. Chem. and Technol., 3, 307-314.

Belgrader, P., Ness, K., Pourahmadi, P., Brounstein, K., Northrup, M.A., Long, G., 2002. A rapid, flow-through, DNA extraction module for integration into microfluidic systems. In: Baba, Y., Shoji., S., van den Berg, A. (Eds.), Micro Total Analysis Systems 2002. Kluwer Academic, Netherlands, pp. 697-698.

Belgrader, P., Elkin, C.J., Brown, S.B., Nasarabadi, S.N., Milanovich, F.P., Colston, B.W., Marshall, G.D., 2003. A reusable flow-through polymerase chain reaction instrument for the continuous monitoring of infectious biological agents. Anal. Chem., 75, 3446-3450.

Cheng, Y.S., Barr, E.B., Fan, B.J., Hargis, P.J., Rader, D.J., O'Hern, T.J., Torczynski, J.R., Tisone, G.C., Preppernau, P.L., Young, S.A., Radloff, R.J., 1999. Detection of bioaerosols using multiwavelength UV fluorescence spectroscopy. Aerosol Sci. Technol., 30, 186-201.

Donlon, M., Jackman, J., 1999. DARPA integrated chemical and biological detection system. Johns Hopkins APL Tech. Dig., 20, 320-325.

Dzenitis, J. M., Hindson, B. J., McBride, M. T., Makarewicz, A. J., Henderer, B. D., Sathyam, U. S., Nasarabadi, S. L., Smith, S. M., Gutierrez, D. M., Metz, T. M., Masquelier, D. M., Brown, S. B., Erler, A. M., Venkateswaran, K. S., Colston, B. W., 2003. The Autonomous Pathogen Detection System, paper presented at the 23rd International Symposium on the Separation of 
Proteins, Peptides, and Polynucleotides, Delray Beach, Florida, USA, 10 Nov 2003, UCRLPRES-200795.

Eitzen, E., Pavlin, J., Cieslak, T., Christopher, G., Culpepper, R., 1999. Medical management of biological casualties. US Army Medical Research Institute of Infectious Diseases, Fort Detrick, pp.11-13.

Fitch, J.P., Gardner, S,N., Kuczmarski, T.A., Kurtz, S., Myers, R., Ott, L.L., Slezak, T.R., Vitalis, E.A., Zemla, A.T., McCready, P.M., 2002. Rapid development of nucleic acid diagnostics. Proc. IEEE, 90, 1708-1721.

Fitch, J.P., Raber, E., Imbro, D.R., 2003. Technology challenges in responding to biological or chemical attacks in the civilian sector. Science, 302, 1350-1354.

Franz D.R, Jahrling, P.B., Friedlander, A.M., McClain, D.J., Hoover, D.L., Bryne, W.R., Pavlin, J.A., Christopher, C.W., Eitzen, E.M., 1997. Clinical recognition and management of patients exposed to biological warfare agents. JAMA, 278, 399-411.

Hilleman, M.R., 2002. Overview: Cause and prevention in biowarfare and bioterrorism. Vaccine, $20,3055-3067$.

Hindson, B.J., Brown, S.B., Marshall, G.D., McBride, M.T., Makarewicz, A.J., Gutierrez, D.M., Wolcott, D.K., Metz, T.R, Madabhushi, R.S., Dzenitis, J.M. and Colston, Jr, B.W., 2004. Development of an automated sample preparation module for environmental monitoring of biowarfare agents. Anal. Chem., 76, 3492-3497.

Hindson, B.J., McBride, M.T., Makarewicz, A.J., Henderer, B.D., Setlur, U.S., Smith, S.M., Gutierrez, D.M., Metz, T.R., Nasarabadi, S.L., Venkateswaran, K.S., Farrow, S.W., Colston, Jr., B.W., Dzenitis, J.M., 2004. Autonomous detection of aerosolized biological agents by multiplexed immunoassay with PCR confirmation. Anal. Chem., submitted. 
Lenehan, C. E., Barnett, N.W., Lewis, S.W, 2002. Sequential injection analysis- Review. Analyst, $127,997-1020$.

Lester, E.D., Ponce, A., 2002. An anthrax "smoke" detector. IEEE Engineering in Medicine \& Biology Magazine, 21, 38-42.

Ligler, F.S., Anderson, G.P., Davidson, P.T., Foch, R.J., Ives, J.T., King, K.D., Page, G., Stenger, D.A., Whelan, J.P., 1998. Remote sensing using an airborne biosensor. Environ. Sci. Technol., 32, 2461-2466.

Luoma, G.A., Cherrier, P.P., Retfalvi, L.A., 1999. Real-time warning of biological-agent attacks with the Canadian Integrated Biochemical Agent Detection System II (CIBADS II). Field Analyt. Chem. Technol., 3, 260-273.

Makino, S.L., Cheun, H.I., Watari, M., Uchida,I., Takoshi, K., 2002. Detection of anthrax spores from the air by real-time PCR. Lett. Appl. Microbiol., 33, 237-240.

McBride, M.T., Gammon, S., Pitesky, M., O’Brien, T.W., Smith, T., Aldrich, J., Langlois, R., Venkateswaran, K.S., 2003. Multiplexed liquid arrays for simultaneous detection of simulants of biological warfare agents. Anal. Chem., 75, 1924-1930.

McBride, M.T., Masquelier, D., Hindson, B.J., Makarewicz, A.J., Brown, S., Burris, K., Metz, T., Langlois, R.G., Tsang, K-T., Bryan, R., Anderson, D.O., Venkateswaran, K.S., Milanovich, F.P., Colston, B.W, 2003. Autonomous detection of aerosolized Bacillus anthracis and Yersinia pestis. Anal. Chem., 75, 5293-5299.

Nasarabadi, S., Milanovich, F.P., Richards, J., Belgrader, P., 1999. Simultaneous detection of Taqman probes containing Fam and Tamra reporter fluorophores. Biotechniques, 27, 11161119. 
Ryzhov, V., Hatout, Y., Fensalau, C., 2000. Rapid characterization of spores of Bacillus cereus group bacteria by matrix-assisted laser desorption-ionization time-of-flight mass spectrometry. Appl. Environ. Microbiol., 66, 3828-3834.

Sincock, S.A., Kulaga, H., Cain, M., Anderson, P., Stopa, P.J, 1999. Applications of flow cytometry for the detection and characterization of biological aerosols. Field Analyt. Chem. Technol., 3, 291-306.

Snyder, A.P., Maswadeh, W.M., Parsons, J.A., Tripathi, A., Meuzelaar, H.L.C., Dworzanski, J.P., Kim, M-G., 1999. Field detection of Bacillus spore aerosols with stand-alone pyrolysis-gas chromatography-ion mobility spectrometry. Field Analyt. Chem. Technol., 3, 315-326.

Stratis-Cullum, D.N., Griffin, G.D., Mobley, J., Vass, A.A., Vo-Dinh, T., 2003. A miniature biochip system for detection of aerosolized Bacillus globigii spores. Anal. Chem., 75, 275-280.

Visuri, S.R., Ness, K.D., Dzenitis, J.M., Benett, B., Bettencourt, K., Hamilton, J., Fisher, K., Krulevitch, P., 2002. Microfluidic tools for biological sample preparation. $2^{\text {nd }}$ Annual International Meeting of IEEE, Engineers in Medicine and Biology Society Special Topic Conference on Microtechnologies and Biology, Madison, Wisconsin, May 2-4. 